\title{
ON ORBITS ASSOCIATED WITH SYMMETRIC SPACES
}

\author{
BY BERTRAM KOSTANT AND STEPHEN RALLIS
}

Communicated by Gian-Carlo Rota, March 27, 1969

1. Introduction. The results of [2] on the orbit structure of a complex reductive Lie algebra, under the adjoint group, are generalized to the analogue associated with a symmetric space. Notwithstanding some new complications such as the nonirreducibility of the variety of nilpotent elements and the nonnormality of orbits of maximal dimension the symmetric space case seems to be the correct setting for the results.

The notions of the principal TDS, principal nilpotent element, regular element, the finiteness of the number of nilpotent orbits and the orbit structure as described by the invariant polynomials and nilpotent orbits all go through in the general case. Also (see [3]) the orbits of maximal dimension can be sectioned in an as nice a manner as in the case of the adjoint representation (see [2]).

2. Regular, semisimple and nilpotent elements.

2.1. Let $g$ be a complex reductive Lie algebra and let $g_{R}$ be a real form fixed throughout. Also let $\mathfrak{g}_{R}=\mathfrak{l}_{R}+\mathfrak{p}_{R}$ be a Cartan decomposition of $\mathfrak{g}_{R}$ fixed throughout. Thus exp ad $\mathfrak{f}_{R}$ operating on $\mathfrak{g}_{R}$ is a maximal compact subgroup of the adjoint group of $g_{R}$ and if

$$
\mathfrak{g}=\mathfrak{l}+\mathfrak{p}
$$

is the linear direct sum obtained by complexifying $\mathfrak{l}_{R}$ and $\mathfrak{p}_{R}$ then the $\operatorname{map} \theta: \mathfrak{g} \rightarrow \mathfrak{g}$ defined by putting $\theta=1$ on $\mathfrak{f}$ and -1 on $\mathfrak{p}$, is a Lie algebra automorphism of order 2 .

Now let $G$ be the adjoint group of $g$ and let $K_{\theta}$ be the subgroup of elements $a \in G$ which commute with $\theta$. Thus $\mathfrak{l}$ and $\mathfrak{p}$ are stable under the action of $K_{\theta}$ and in particular the latter defines a representation

$$
K_{\theta} \rightarrow \text { Aut p }
$$

which will be of concern to us throughout. One notes of course that the identity component $K$ of $K_{\theta}$ is just the subgroup of $G$ corresponding to $t$.

Let $\mathfrak{a}_{R}$ be a maximal abelian subalgebra of $\mathfrak{p}_{R}$ and let $\mathfrak{a} \subseteq \mathfrak{p}$ be its complexification. Let $A=\exp$ ad $\mathfrak{a}$ and let $F$ be the finite group of all elements of order 2 in $A$. Clearly $F \subseteq K_{\theta}$ and hence $F$ normalizes $K$. The relation between $K_{\theta}$ and $K$ is clarified by

Proposition 1. One has $K_{\theta}=F K$. 
An element $x \in \mathfrak{g}$ is called semisimple if ad $x$ is diagonalizable and is called nilpotent if $x \in[\mathfrak{g}, \mathfrak{g}]$ and ad $x$ is nilpotent. One knows that any $x \in \mathfrak{g}$ may be uniquely written (Jordan decomposition) $x=x_{2}+x_{n}$ where $\left[x_{s}, x_{n}\right]=0$ and $x_{s}$ is semisimple and $x_{n}$ nilpotent. In case $x \in \mathfrak{p}$ it is immediate that both $x_{s}, x_{n} \in \mathfrak{p}$. Let $\delta$ and $\Re$ be respectively the set of all semisimple and nilpotent elements in $\mathfrak{p}$. One may characterize the elements of $\delta$ by

Proposition 2. Let $x \in p$ then $x \in S$ if and only if it is $K$ (or $K_{\theta}$ ) conjugate to an element in $a$.

2.2. If $f$ is a function on $p$ and if $a \in K_{\theta}$ then $a \cdot f$ is the function on $p$ defined by $(a \cdot f)(x)=f\left(a^{-1} \cdot x\right)$. If $S^{\prime}$ denotes the ring of all polynomial functions on $p$ then in this way clearly $S^{\prime}$ is a $K_{\theta}$-module. Let $J^{\prime}=\left(S^{\prime}\right)^{K_{\theta}}$ be the subring of $K_{\theta}$ invariants and let $J_{+}^{\prime}$ be the ideal of all $f \in J^{\prime}$ which vanish at the origin (that is with zero as a constant term). The set of nilpotent elements in $\mathfrak{p}$ is characterized by

Proposition 3. Let $x \in \mathfrak{p}$ then $x \in \mathfrak{N}$ if and only if $f(x)=0$ for all $\in J_{+}^{\prime}$.

For any $x \in p$ the connected components of the orbit $K_{\theta} \cdot x$ all have the same dimension (written $\operatorname{dim} K_{\theta} \cdot x$ ). An element $x \in \mathfrak{p}$ is called regular in case $\operatorname{dim} K_{\theta} \cdot x \geqq \operatorname{dim} K_{\theta} \cdot y$ for any $y \in \mathfrak{p}$. That is, in case $\operatorname{dim} \mathfrak{l}^{x} \leqq \operatorname{dim} \mathfrak{q}^{y}$ for all $y \in \mathfrak{p}$. If $\dot{z} \subseteq g$ is any subset $\mathbb{8}^{x}$ denotes the subset of all elements in 8 which commute with $x$. Let $R$ be the set of all regular elements in $\mathfrak{p}$. Clearly $R$ and $\delta$ are dense in $\mathfrak{p}$.

Let $r=\operatorname{dim} a$. Then if $\operatorname{codim} K_{\theta} \cdot x=\operatorname{dim} \mathfrak{p}-\operatorname{dim} K_{\theta} \cdot x$ one can show that for any $x \in \mathfrak{p}$

$$
\operatorname{codim} K_{\theta} \cdot x=\operatorname{dim} \mathfrak{p}^{x} \text { and } r \leqq \operatorname{dim} \mathfrak{p}^{x}
$$

where equality holds if and only if $x \in R$.

2.3. Any Lie subalgebra of $g$ which is isomorphic to the Lie algebra of $S L(2, \boldsymbol{C})$ is called a TDS. A TDS $\mathfrak{u}$ is called normal in case $\mathfrak{u}$ is stable under $\theta$ and $\mathfrak{u} \Phi \mathfrak{l}$. It is immediate that if $\mathfrak{u}$ is a normal TDS then $\mathfrak{u} \cap \mathfrak{l}$ is 1 -dimensional and $\mathfrak{u} \cap \mathfrak{p}$ is 2 -dimensional. An $S$-triple is a set of 3-linear independent elements $(x, e, f)$ in $g$ where $[x, e]=2 e$, $[x, f]=-2 f$ and $[e, f]=x$. It is called normal in case $e, f \in \mathfrak{p}$ and $x \in \mathfrak{l}$. It is clear that an $S$-triple spans a TDS and a normal $S$-triple spans a normal TDS. Strengthening the Jacobson-Morosov theorem and Corollary 3.7 of $[1]$ one has

Proposition 4. Any $0 \neq e \in \mathfrak{T}$ can be embedded in a normal S-triple $(x, e, f)$ (and hence in a normal TDS). Moreover this sets up a 1-1 corre- 
spondence between the set of all $K_{\theta}$-orbits in $\Re-(0)$ and all $K_{\theta}$-conjugacy classes of S-triples.

Using the ideas of $\S 4$ in [1] one shows the $K_{\theta}$-conjugacy class of a normal $S$-triple $(x, e, f)$ is determined by the $K_{\theta}$-conjugacy class of $x$. It then follows easily that there are only a finite number of $K_{\theta}$-orbits in $\Re$. This implies that each Zariski component of $\Re$ in $\mathfrak{p}$ has codimension $r$. But more than this one has

THEOREM 1. The set $R \cap \Re$ is open and dense in $\Re$. Moreover $R \cap \Re$ is a single $K_{\theta}$-orbit. Thus $R \cap \Re$ may be characterized as the unique open $K_{\theta}$-orbit in $\Re$ or as the unique dense orbit in $\Re$.

REMARK 1 . Unlike the situation in [1] $\Re$ is not necessarily Zariski irreducible. Note that Theorem 1 implies that the topological components of $R \cap \Re$ are the same as the Zariski components of $R \cap \Re$ and that all have the same dimension, all are open in their closures and that the closures are just the Zariski components of $\Re$.

2.4. The elements of $R \cap \Re$ are called principal nilpotent. A normal $S$-triple $(x, e, f)$ is called principal if $e$ (and hence $f$, and vice versa) is principal nilpotent. A normal TDS $\mathfrak{u}$ is called principal in case $\mathfrak{u}$ contains a principal nilpotent element, in which case all the nonzero nilpotent elements in $\mathfrak{u}$ are principal.

Corollary to Theorem 1. Any 2 principal normal $S$-triples or principal normal TDS are $K_{\theta}$-conjugate.

The principal nilpotent elements in $\mathfrak{p}$ are related to certain nilpotents in $g_{R}$. Let $G_{R}$ be the subgroup of all elements $a \in G$ which leave $\mathfrak{g}_{R}$ stable. Let $\mathfrak{L}$ be the set of all nilpotent elements in $\mathfrak{g}$ and let $\mathfrak{L}_{R}=\mathfrak{L} \cap \mathrm{g}_{R}$. The action of $G$ on $\mathscr{L}$ is described in [1]. With regard to the action of $G_{R}$ on $\mathscr{L}_{R}$ one has

Theorem 2. There exists a unique dense $G_{R}$ orbit $P$ in $\mathfrak{L}_{R}$. Moreover $P$ is open in $\mathfrak{L}_{R}$. An element $e \in \Re$ is principal nilpotent if and only if

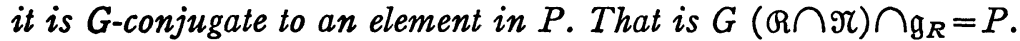

2.5. Let $\Lambda \subseteq \mathfrak{a}^{\prime}$ be the set of roots (that is restricted roots in the usual terminology) for the action of $\mathfrak{a}$ on $\mathfrak{g}$ and let $\Sigma \subseteq \Lambda$ be the set of simple roots relative to some lexicographical ordering. For any $\phi \in \Lambda$ let $\mathfrak{g}^{\phi} \subseteq \mathfrak{g}$ be the corresponding root space.

Another characterization of principal nilpotent is given in

TheORem 3. Let $G^{A}$ be the centralizer of $A$ in G. Then $\sum_{\alpha \in \Sigma} \mathfrak{g}^{\alpha}=\mathfrak{g}^{1}$ is stable under $G^{A}$ and there exists a unique open dense orbit $D$ of $G^{A}$ in 
$\mathfrak{g}^{1}$. An element $e \in \mathfrak{p}$ is principal nilpotent if and only if it is G-conjugate to an element in $D$.

2.6. Now the set $R$ of regular elements can be characterized in terms of the notion of principal nilpotent. Let $x \in \mathfrak{p}$ be arbitrary and let $x=x_{s}+x_{n}$ be its Jordan decomposition. Now $\mathrm{g}^{x_{s}}$ is reductive and $\mathfrak{g}^{x_{s}}=\mathfrak{f}^{x_{s}}+\mathfrak{p}^{x_{s}}$ is a complexified Cartan decomposition relative to a suitable real form of $g^{x_{a}}$. Thus the theory above applies to this case and in particular we can speak of the principal nilpotent elements in $\mathfrak{p}^{x_{s}}$. One notes of course that $x_{n}$ is a nilpotent element in $\mathfrak{p}^{x_{a}}$.

PROPOSITION 5. Let $x \in \mathfrak{p}$ be arbitrary. Then $x$ is regular if and only if $x_{n}$ is principal nilpotent in $\mathfrak{p}^{x_{4}}$.

\section{The orbit structure.}

3.1. Now the ring $J^{\prime}=\left(S^{\prime}\right)^{K_{\theta}}$ of polynomial $K_{\theta}$ invariants, by Chevalley, is a polynomial ring in $r(=\operatorname{dim} \mathfrak{a})$ homogeneous generators $u_{1}, \cdots, u_{r}$. This defines a map

$$
u: \mathfrak{p} \rightarrow \boldsymbol{C}^{r}
$$

by $u(x)=\left(u_{1}(x), \cdots, u_{r}(x)\right)$. Also if $\mathcal{O}$ is the set of all $K_{\theta}$-orbits in $\mathfrak{p}$ then since $u$ is constant on any orbit it induces a map $\bar{u}: \mathcal{O} \rightarrow \boldsymbol{C}^{r}$. Let $\theta_{s}$ be the set of all orbits of semisimple elements. They are all characterized as follows:

TheOREM 4. An orbit $O \in O$ is closed (either Zariski or in the usual topology) if and only if $O \in \Theta_{S}$.

Let $\boldsymbol{\theta}_{R}$ be the set of all orbits of regular elements. By definition $\boldsymbol{\theta}_{R}$ is the set of all orbits of maximal dimension (those orbits having codimension $r$ ). Let $\bar{u}_{S}$ and $\bar{u}_{R}$ be respectively the restrictions of $\bar{u}$ to $\hat{\theta}_{S}$ and $\boldsymbol{\theta}_{R}$.

Theorem 5. The map $\bar{u}_{S}: \theta_{S} \rightarrow C^{r}$ and $\bar{u}_{R}: \theta_{R} \rightarrow C^{r}$ are both bijections.

3.2. For any $\xi \in C^{r}$ let $O_{S}(\xi)=\bar{u}_{S}^{-1}(\xi)$ and $O_{R}(\xi)=\bar{u}_{R}^{-1}(\xi)$. Now obviously $\mathfrak{p}=\bigcup_{\xi \in C^{r}} u^{-1}(\xi)$ is a disjoint union and each $u^{-1}(\xi)$ is nonempty and stable under the action of $K_{\theta}$. As a generalization of Theorem 3 in [2] one has

TheOREM 6. For any $\xi \in C^{r}$ there are only a finite number of $K_{\theta}$-orbits in $u^{-1}(\xi)$. Moreover, there is a unique orbit of maximal dimension and $a$ unique orbit of minimal dimension in $u^{-1}(\xi)$. These are respectively $O_{R}(\xi)$ and $O_{S}(\xi)$. Moreover, $O_{R}(\xi)$ is open and dense in $u^{-1}(\xi)$ (implying that all Zariski components of $u^{-1}(\xi)$ have codimension $\left.r\right)$. Also $O_{S}(\xi)$ is irreducible and is the unique closed orbit in $u^{-1}(\xi)$. 
Considering $G$-conjugacy (as far as it goes) in $\mathfrak{p}$ instead of $K_{\theta}$ conjugacy does not enlarge an orbit of regular or semisimple elements.

THEOREM 7. Let $x, y \in \mathfrak{p}$. Then if $x$ is semisimple or regular $x$ and $y$ are $K_{\theta}$-conjugate if and only if they are $G$-conjugate. If $x$ is semisimple we may substitute $K$ for $K_{\theta}$.

\section{REFERENCES}

1. Bertram Kostant, The principal three dimensional subgroup and the Betti numbers of a complex simple Lie group, Amer. J. Math. 81 (1959), 973-1032.

2. - Lie group representations on polynomial rings, Amer. J. Math. 86 (1963), 327-402.

3. Bertram Kostant and Stephen Rallis, On representations associated with symmetric spaces, Bull. Amer. Math. Soc. 75 (1969), 884-888.

Massachusetts Institute of Technology, Cambridge, Massachusetts 02139 